\title{
Malnutrition and depression in the institutionalised elderly
}

\author{
Christine Smoliner ${ }^{1}$, Kristina Norman ${ }^{1}$, Karl-Heinz Wagner ${ }^{2}$, Wolfgang Hartig ${ }^{3}$, Herbert Lochs ${ }^{1}$ \\ and Matthias Pirlich ${ }^{1}$ \\ ${ }^{1}$ Department of Gastroenterology, Hepatology and Endocrinology, Charité Universitätsmedizin Berlin, Charitéplatz, 1, \\ 10117 Berlin, Germany \\ ${ }^{2}$ Department of Nutritional Sciences, University of Vienna, Althanstrasse 14, 1090 Vienna, Austria \\ ${ }^{3}$ Klinikum St. Georg gGmbH, Delitzscher Straße 141, 04129 Leipzig, Germany
}

(Received 8 January 2009 - Revised 25 May 2009 - Accepted 1 June 2009 - First published online 22 July 2009)

Malnutrition and depression are highly prevalent in the institutionalised elderly and can lead to unfavourable outcomes. The aim of the present study was to assess associations between nutritional status and depressive symptoms and to explore their impact on self-caring capacity and quality of life (QoL) in elderly nursing-home residents (NHR). We conducted a cross-sectional study with 114 NHR (eighty-six female) with a mean age of 84.6 (SD 9.1) years. Nutritional status was assessed with the Mini Nutritional Assessment (MNA). Depressive symptoms were rated with the Geriatric Depression Scale (GDS). Self-caring capacity was measured with the Barthel index (BI) and QoL was assessed with the short-form thirtysix-item (SF-36) questionnaire. Of the NHR, twenty-six (22.8\%) were malnourished according to the MNA and sixty-six (57.9\%) were at nutritional risk. Of the residents, seventy-five could be assessed with the GDS, whereof sixteen $(21.3 \%)$ had major and twenty-six (34.7\%) had minor depressive symptoms. GDS scores tended to be higher in patients with impaired nutritional status (5.4 (SD 3.6) in well-nourished subjects and 6.9 (SD 3.2) in residents with malnutrition or at risk of malnutrition). The MNA correlated significantly with the GDS $(r-0 \cdot 313 ; P=0 \cdot 006)$ and the GDS emerged as the only independent risk factor for malnutrition in a multiple regression analysis, whereas age, sex, care level, number of prescriptions and self-caring capacity had no influence. The BI was not reduced in patients with a high GDS. QoL was affected in malnourished residents as well as in study participants with depressive symptoms. The results of the present study point towards an association between malnutrition and depressive symptoms. However, the relationship is complex and it remains unclear whether depression in NHR is the cause or consequence of impaired nutritional status. Further studies are needed to identify the direction of this relationship and to assess the effect of depression treatment on nutritional and functional status as well as on QoL.

Nursing-home residents: Malnutrition: Depression: Quality of life

Malnutrition is a frequent problem in aged individuals. Whereas the prevalence in free-living elderly ranges between 5 and $10 \%(1)$, it can reach up to $60 \%$ in institutionalised individuals $^{(2,3)}$. The aetiology of malnutrition is multifactorial and the consequences are severe: a decrease in functional status ${ }^{(4)}$ and consequently high dependency, an impaired quality of life ${ }^{(5)}$ as well as higher morbidity and mortality ${ }^{(6,7)}$.

Depression is a further common health problem in late life with prevalence rates of up to $45 \%$ in the institutionalised elderly ${ }^{(8-10)}$. The consequences of untreated depression are manifold and cause an increased use of health care and medical services ${ }^{(9,11)}$, affect quality of life and well-being negatively ${ }^{(11)}$ and can cause increased mortality ${ }^{(12)}$. Despite growing evidence that depression is a problem especially in the institutionalised elderly, only little attention is paid to this fact ${ }^{(10)}$. Depression very often remains undiagnosed and therefore untreated ${ }^{(9,10)}$.

The causal relationship between depression and nutritional status is still unclear. Several studies indicate that depression is a major factor contributing to weight loss in the elderly ${ }^{(13,14)}$. While depression is associated with weight gain in individuals before age 50 years, it leads to weight loss after that age, as was observed in a cohort of US adults $^{(15)}$. Moreover, studies in hospitalised and communitydwelling elderly showed that depression increased the risk of impaired nutritional status and observed that malnourished patients had higher depression scores ${ }^{(16,17)}$.

The aim of the present study was to assess nutritional status and prevalence of depression and associations between both. Furthermore, we wanted to explore their impact on selfcaring capacity and quality of life in elderly nursing-home residents.

\section{Methods}

A total of 114 out of 140 residents of a nursing home in Berlin (Germany) were included in the present study. The residents or their legal custodian respectively were asked to give written

Abbreviations: GDS, Geriatric Depression Scale; MNA, Mini Nutritional Assessment; SF-36, short-form thirty-six question.

* Corresponding author: Christine Smoliner, fax +44 207188 6761, email christine.smoliner@charite.de 
informed consent for participation. All questionnaires were administered in face-to-face interviews. Information on diagnosis and medication was taken from medical documentation and cognitive status was rated by the nursing-home staff by clinical judgement.

The study was approved by the ethics committee of the Charité Universitätsmedizin Berlin, Campus Mitte and was conducted according to the guidelines of the declaration of Helsinki.

Nutritional status was assessed with the Mini Nutritional Assessment (MNA). The MNA is a validated nutrition screening and assessment tool to identify geriatric patients who are malnourished or at risk of malnutrition ${ }^{(18)}$. It includes questions on general condition, appetite, anthropometry, food preferences and subjective perception of health status. The MNA could be obtained from all participants, since the nursing staff was interviewed about subjects with cognitive impairment or dementia as recommended by Vellas et al. ${ }^{(18)}$. A normal nutritional status is indicated with greater or equal to 24 points on a 30 -point scale, 17 to 23.5 points imply risk of malnutrition and subjects with less than 17 points are considered malnourished.

Body weight was measured in light clothes with an electronic chair scale (Seca 959; Seca, Hamburg, Germany) or a bed scale (Bolero; Arjo, Gloucester, UK) to the nearest $0 \cdot 1 \mathrm{~kg}$. To calculate weight loss, weight measurements taken during the preceding 3 months were obtained from medical documentation. Body height was taken with a measuring rod (Seca 220; Seca) to the nearest $0.1 \mathrm{~cm}$. If height could not be elicited this way, it was calculated from knee height according to the formula by Chumlea et al. ${ }^{(19)}$.

BMI was calculated with the formula: weight $(\mathrm{kg}) /$ height $\left(\mathrm{m}^{2}\right)$

Mid-upper arm circumference was measured with a nonelastic tape on the non-dominant relaxed arm midway between the tip of the acromion and the olecranon process. In individuals with paralysed or severely handicapped extremities, measures where taken from the healthy extremity. Calf circumference was measured with a non-elastic tape on the thickest part of the undressed calf, with the individual sitting and having their knee flexed at a $90^{\circ}$ angle.

The Geriatric Depression Scale (GDS) is a screening instrument to identify patients at risk of depression. The short-form used in the present study consists of fifteen yes/no questions. A score of 0 to 5 points is considered normal, more than 5 points indicates mild depression and more than 10 points indicates severe depression ${ }^{(20)}$. The GDS is suitable for healthy, medically ill and mild to moderately cognitively impaired older adults ${ }^{(21)}$.

Functional status was assessed with the Barthel index, which is an instrument to systematically evaluate the selfcaring capacity of older adults. The individual's ability to independently perform basic activities of daily living such as eating, body care and mobility is measured ${ }^{(22)}$.

Quality of life was assessed with the validated Medical Outcomes Study thirty-six-item Short-Form General Health Survey ${ }^{(23)}$. The questionnaire consists of thirty-six questions assessing quality of life and well-being in eight multi-item scales. The components 'mental health', 'role emotional' and 'social functioning' assess anxiety and the impact of emotional problems on daily life and social contacts. 'Vitality' and 'general health' display the personal perception of the global health status. 'Physical functioning', 'role physical' and 'bodily pain' measure physical ability and limitations in daily life arising due to decreased functional status and pain.

Statistical analysis was carried out with the software package SPSS (version 15; SPSS Inc., Chicago, IL, USA). All data are shown as mean values and standard deviations unless indicated otherwise. The Mann-Whitney $U$ test was used for comparison between groups due to slightly skewed data and the small sample size. The $\chi^{2}$ test was performed for comparison of nominal variables. Spearman's correlation was calculated to assess the relationship between variables. We performed multiple stepwise regression analysis with the MNA and GDS as dependent variables to identify factors influencing nutritional status and depression. An acceptable level of statistical significance was established a priori at $P<0.05$.

\section{Results}

The mean age of the study population was 84.6 (SD 9.1) years and three-quarters of the study participants were female ( $n$ 86). Residents with high dependency on nursing and care (care level 2 and greater) represented $63.2 \%$ of the study population.

Of the nursing-home residents, twenty-six $(22 \cdot 8 \%)$ were malnourished, sixty-six $(57.9 \%)$ were at risk of malnutrition and twenty-two $(19 \cdot 3 \%)$ were well-nourished according to the MNA. Table 1 shows the demographic and nutritional parameters of the study population according to nutritional status.

The GDS was administered to seventy-five study participants. A total of twenty-six residents $(34.7 \%)$ met criteria for mild depression, whereas sixteen $(21.3 \%)$ showed signs

Table 1. Subject characteristics according to nutritional status (includes only study participants that could be assessed with the Geriatric Depression Scale)

(Mean values and standard deviations)

\begin{tabular}{|c|c|c|c|c|}
\hline & \multicolumn{2}{|c|}{$\begin{array}{l}\text { Well- } \\
\text { nourished } \\
\text { (MNA } \\
\text { group 1) }\end{array}$} & \multicolumn{2}{|c|}{$\begin{array}{l}\text { Malnourished } \\
\text { or at risk of } \\
\text { malnutrition } \\
\text { (MNA groups } \\
2 \text { and } 3+\text { ) }\end{array}$} \\
\hline & Mean & SD & Mean & SD \\
\hline Number of subjects & \multicolumn{2}{|c|}{21} & \multicolumn{2}{|c|}{54} \\
\hline Men & \multicolumn{2}{|c|}{10} & \multicolumn{2}{|c|}{14} \\
\hline Women & \multicolumn{2}{|c|}{11} & \multicolumn{2}{|c|}{40} \\
\hline Age (years) & $81 \cdot 3$ & $10 \cdot 1$ & 83.9 & $8 \cdot 6$ \\
\hline Weight (kg) & $75 \cdot 2$ & $16 \cdot 9$ & $60 \cdot 1^{*}$ & $11 \cdot 8$ \\
\hline $\mathrm{BMI}\left(\mathrm{kg} / \mathrm{m}^{2}\right)$ & $27 \cdot 6$ & $6 \cdot 1$ & $22 \cdot 9^{*}$ & 4.4 \\
\hline Weight loss (\%) & $4 \cdot 6$ & $3 \cdot 2$ & $8 \cdot 9$ & $5 \cdot 2$ \\
\hline Geriatric Depression Scale (points) & 5.4 & 3.6 & $6 \cdot 9$ & $3 \cdot 2$ \\
\hline Barthel index (points) & 71.9 & $23 \cdot 3$ & $61 \cdot 6^{*}$ & $26 \cdot 6$ \\
\hline \multicolumn{5}{|l|}{ Length of stay (years) } \\
\hline Median & \multicolumn{2}{|c|}{$1 \cdot 0$} & \multicolumn{2}{|c|}{0.9} \\
\hline Interquartile range & \multicolumn{2}{|c|}{$0.6-3.4$} & \multicolumn{2}{|c|}{$0.5-2.8$} \\
\hline Care level & $1 \cdot 4$ & 0.7 & $1 \cdot 5^{*}$ & 0.6 \\
\hline Number of drugs & $4 \cdot 8$ & $2 \cdot 0$ & 5.4 & $2 \cdot 5$ \\
\hline
\end{tabular}

MNA, Mini Nutritional Assessment.

${ }^{\star}$ Mean value was significantly different from that of the well-nourished group $(P<0.05)$.

† Groups 2 and 3 were pulled together due to small sample size. 
Table 2. Subject characteristics according to evaluation by the Geriatric Depression Scale (GDS)

(Mean values and standard deviations)

\begin{tabular}{|c|c|c|c|c|c|}
\hline & \multicolumn{2}{|c|}{$\begin{array}{l}\text { Residents } \\
\text { rated by the } \\
\text { GDS }\end{array}$} & \multicolumn{2}{|c|}{$\begin{array}{l}\text { Residents not } \\
\text { rated by the } \\
\text { GDS }^{*}\end{array}$} & \multirow[b]{2}{*}{$P$} \\
\hline & Mean & SD & Mean & SD & \\
\hline Number of subjects & \multicolumn{2}{|c|}{75} & \multicolumn{2}{|c|}{39} & \\
\hline Men & \multicolumn{2}{|c|}{24} & \multicolumn{2}{|c|}{4} & \\
\hline Women & \multicolumn{2}{|c|}{51} & \multicolumn{2}{|c|}{35} & \\
\hline Age (years) & 83.2 & $9 \cdot 1$ & $87 \cdot 3$ & 8.7 & 0.017 \\
\hline BMI $\left(\mathrm{kg} / \mathrm{m}^{2}\right)$ & 24.2 & $5 \cdot 3$ & 21.4 & $5 \cdot 2$ & 0.025 \\
\hline $\begin{array}{l}\text { Mini Nutritional } \\
\text { Assessment (points) }\end{array}$ & $22 \cdot 0$ & $3 \cdot 0$ & $16 \cdot 3$ & $4 \cdot 1$ & $<0.0001$ \\
\hline Barthel index (points) & 64.5 & $26 \cdot 0$ & $19 \cdot 7$ & \multirow[t]{2}{*}{$25 \cdot 0$} & $<0.0001$ \\
\hline Length of stay (years) & & & & NS \\
\hline Median & \multirow{2}{*}{\multicolumn{2}{|c|}{$\begin{array}{c}0.8 \\
0.5-2.1\end{array}$}} & \multirow{2}{*}{\multicolumn{2}{|c|}{$\begin{array}{c}1.2 \\
0.6-4.2\end{array}$}} & \\
\hline Interquartile range & & & & & \\
\hline Care level & 1.4 & 0.7 & $2 \cdot 2$ & 0.7 & $<0.0001$ \\
\hline Number of drugs & $5 \cdot 2$ & $2 \cdot 4$ & $4 \cdot 1$ & $2 \cdot 1$ & 0.011 \\
\hline
\end{tabular}

${ }^{*}$ Residents with severe cognitive impairment were excluded from GDS evaluation

of severe depression according to the GDS. Only thirty-three $(44 \%)$ scored less than five points and were considered being without depression. GDS scores did not differ between men and women. A comparison of depressive and nondepressive elderly did not reveal significant differences in age, length of stay, care level, BMI, daily number of prescriptions or Barthel index (data not shown). Subjects who could not be assessed with the GDS due to cognitive impairment were older, had a significantly worse nutritional status and a significantly lower Barthel index (see Table 2).

Of the forty-two residents considered to have mild or severe depressive symptoms according to the GDS, only seven had a documented diagnosis of depression in their medical charts. One individual who had been diagnosed depressive in medical documentation had a GDS $\leq 5$.

The GDS and MNA correlated significantly ( $r-0 \cdot 313$; $P=0.006)$ but the MNA was not significantly lower in study participants with depressive symptoms (see Fig. 1). In a multiple regression analysis the GDS emerged as the only independent risk factor for malnutrition $\left(R^{2} 0.089 ; F=7.9\right.$; $P=0.006$ ), while age, sex, care level, number of prescriptions and Barthel index did not. Vice versa, the MNA was the only risk factor significantly associated with the GDS $\left(R^{2} 0 \cdot 089\right.$; $F=7.9 ; P=0.006)$ when entered in an analysis with age, sex, care level, number of prescriptions and Barthel index. In subjects with depressive symptoms, loss of appetite tended to occur more often ( $9 \%$ with GDS $<5$ and $26 \%$ with GDS $>5 ; P=0.059)$, whereas weight loss was not more prevalent $(36 \%$ with GDS $<5$ and $28 \%$ with GDS $>5 ; P=0.478)$.

Quality of life was affected in malnourished residents as shown by significantly lower short-form thirty-six question (SF-36) questionnaire scores in 'general health' $(61 \cdot 3$ (SD 19.4) \% in well-nourished and 50.4 (SD 19.6) \% in malnourished subjects; $P=0.043$ ) and 'vitality' (52.4 (SD 28.7) \% in well-nourished and 37.2 (SD 21.1) \% in malnourished individuals; $P=0.035$ ). Study participants with a GDS $>5$ had significantly reduced 'role physical', 'role emotional', 'general health', 'vitality' and 'mental health' as seen in Fig. 2.

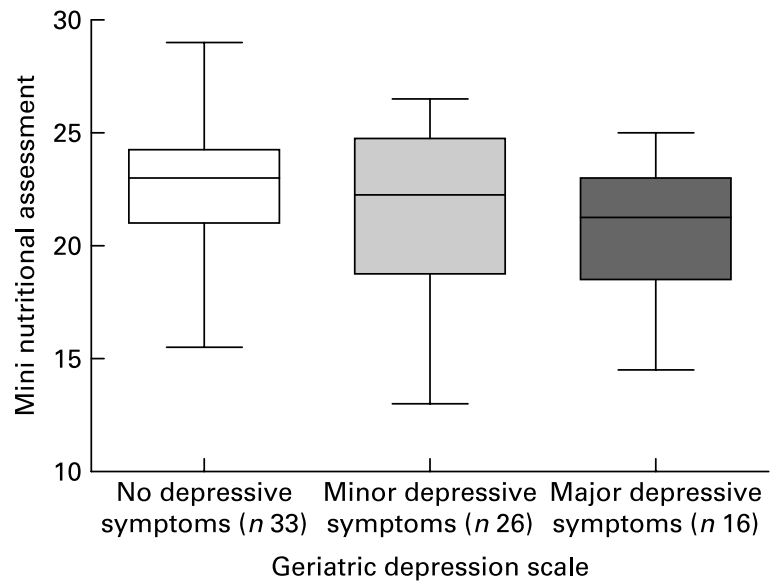

Fig. 1. Nutritional status as assessed by the Mini Nutritional Assessment in patients without depressive symptoms and with minor and major depressive symptoms. The box plots indicate the minimum, the maximum and the 25th, 50th and 75th percentiles. There were no significant differences between the three groups.

\section{Discussion}

In the present study, both malnutrition and depressive symptoms were highly prevalent and showed a modest association. While Barthel index and quality of life were reduced in malnourished subjects, Barthel index was not affected in depressed nursing-home residents. However, perception of physical ability and various components of quality of life were impaired in residents with depressive symptoms.

The high number of individuals identified as malnourished and at nutritional risk is in concordance with the literature ${ }^{(3)}$ as is the number of residents with symptoms of mild or severe depression ${ }^{(8,10)}$. Nutritional status was associated with depressive symptoms as seen by a significant correlation between the MNA and GDS. To further clarify this association, we performed a multiple regression analysis that identified the MNA as the only factor significantly influencing the GDS, while age, sex, care level, number of drugs and Barthel index did not. Vice versa, the GDS was the only factor with significant influence on nutritional status. Our findings are supported by studies carried out in elderly hospitalised and community-dwelling elderly. Cabrera et al. found that the risk of developing depression was four times as high in elderly with nutritional deficits as in those without ${ }^{(17)}$. In a recently published study by German et al. elderly hospitalised patients at risk of malnutrition had significantly higher GDS scores and furthermore, a MNA score below 23.5 points increased the risk for depression by $2^{(16)}$.

It remains difficult to establish a clear causal relationship between nutritional status and depression and this in turn might complicate treatment. However, simply treating depression, which might be the initial cause for weight loss, will probably not completely reverse malnutrition in the majority of the elderly. This is true because of the multifactorial nature of impaired nutritional status in older individuals and in addition to their reduced capacity of restoring their nutritional status ${ }^{(24)}$. However, a study published in 2003 evaluating depression therapy in elderly out-patients found that serotonin reuptake inhibitors affected the nutritional status of non-demented patients positively as seen by weight gain, 


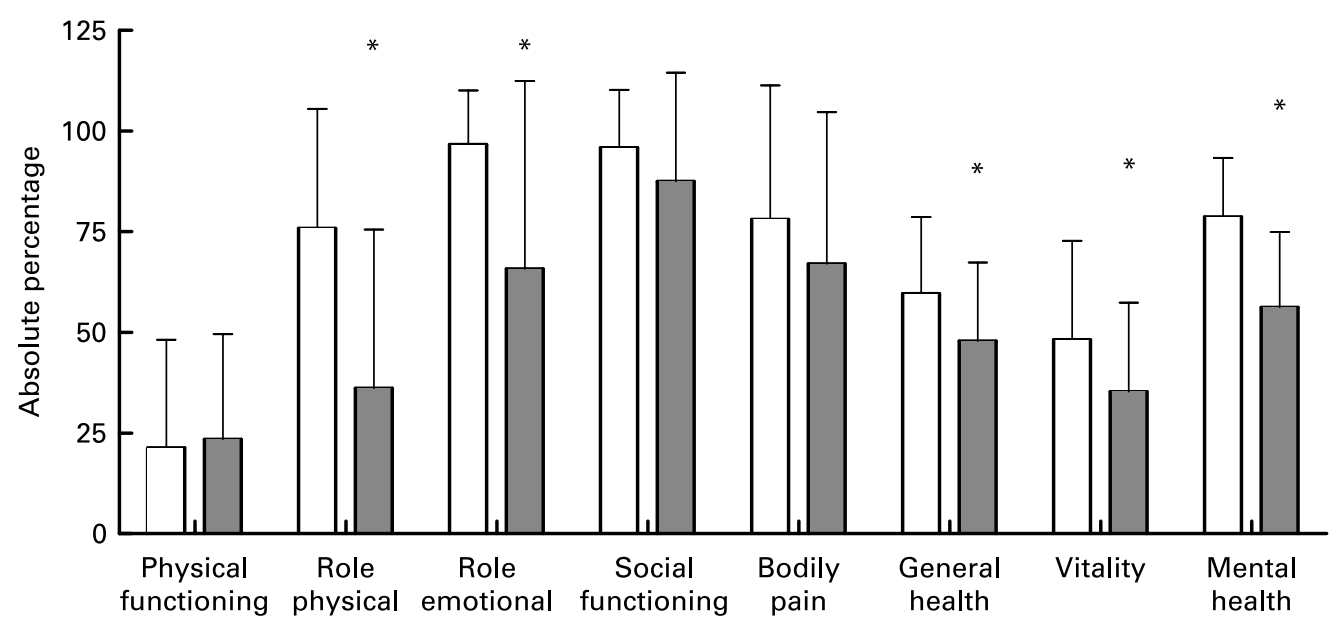

Fig. 2. Nursing-home residents with depressive symptoms (Geriatric Depression Scale $>5$ ) $(\square)$ had lower values in various components of quality of life when compared with residents without depressive symptoms $(\square)$. Values are means, with standard deviations represented by vertical bars. * Mean value was significantly different from that of the group without depressive symptoms: 'role physical', $P<0.0001$; 'role emotional', $P=0.003$; 'general health', $P=0.022$; 'vitality', $P=0.032$; 'mental health', $P<0.0001$.

improved MNA scores and an increase in serum albumin ${ }^{(25)}$. Further intervention studies are needed to clarify the extent of the influence of depression on nutritional status.

In several studies, depression is found to be particularly high among residents with reduced physical activity or impairment in activities of daily living ${ }^{(9,26)}$. In contrast, in the present study the Barthel index was not affected in subjects with minor or severe depressive symptoms. This has been observed in other studies in institutionalised elderly, but also in elderly out-patients with chronic heart failure ${ }^{(11,27)}$. One reason for this could be the fact that individuals admitted to a nursing home or suffering from chronic disease are already impaired in their physical function and their performance of activities of daily living and therefore questionnaires rating physical status might not be sensitive enough to detect changes occurring in depression in this special population ${ }^{(11)}$.

Interestingly, the SF-36 component 'role physical' was significantly reduced with a GDS $>5$. This component reflects an individual's perception of how physical health compromises work or other daily activities. It appears that since the Barthel index and the SF-36 component 'physical functioning' were not reduced in individuals with depressive symptoms, they might not have been as handicapped in actual function as in their perception thereof. This is supported by a study in severely depressed elderly patients, who rated their functionality as impaired whereas their clinicians did not. The authors concluded that the rating by experienced clinicians was able to distinguish disability contributing to depression from disability resulting from medical illness and/or cognitive impairment ${ }^{(28)}$. Another study reported that depressed elderly out-patients with chronic heart failure showed a tendency to underestimate their physical ability, whereas they had similar energy expenditure and walking ability as their non-depressive counterparts ${ }^{(27)}$. As expected, the SF-36 components 'role emotional', 'vitality' and 'mental health' were reduced in depressed residents and, as has been reported in other studies, quality of life was reduced in individuals with impaired nutritional status ${ }^{(5)}$.

In the present study only a small number of participants with a GDS $>5$ had previously been diagnosed with depression in medical documentation. It is known that institutionalised elderly have a three- to four-fold higher probability of falling ill with depression than free-living elderly ${ }^{(8)}$. Yet unrecognised depression and inadequate treatment are common in this population group ${ }^{(12,29)}$. Therefore, depression screening and staff training in depression recognition should be enforced, especially in nursing homes for the elderly ${ }^{(30)}$.

In the present study more than one-third of study participants could not be evaluated with the GDS due to impaired cognitive status. Our data shows that nutritional status, as assessed with BMI and the MNA, was significantly lower in this population. As a prevalence of depression of approximately 20 to $25 \%$ in elderly with dementia is reported in the literature ${ }^{(31)}$ adequate assessment instruments should be employed to explore associations between depression and nutritional status in this vulnerable group ${ }^{(32)}$.

\section{Conclusion}

The results of the present observational study indicate a modest association between depressive symptoms and malnutrition. However, the relationship is complex; it remains unclear whether depression in nursing-home residents is the cause or consequence of impaired nutritional status. Longitudinal studies should be conducted in the nursing home to tackle this question and to assess the effect of depression treatment on nutritional and functional status as well as on quality of life.

\section{Acknowledgements}

The present study received no specific grant from any funding agency in the public, commercial or not-for-profit sectors.

K. N. and M. P. designed the study; C. S. conducted patient recruitment and data collection; C. S., K. N. and M. P. analysed the data and wrote the manuscript; K.-H. W., W. H. and $\mathrm{H}$. L. critically reviewed and edited the manuscript.

The authors do not have any conflict of interest. 


\section{References}

1. Vellas B, Lauque S, Andrieu S, et al. (2001) Nutrition assessment in the elderly. Curr Opin Clin Nutr Metab Care 4, 5-8.

2. Saletti A, Lindgren EY, Johansson L, et al. (2000) Nutritional status according to Mini Nutritional Assessment in an institutionalized elderly population in Sweden. Gerontology 46, $139-145$.

3. Suominen M, Muurinen S, Routasalo P, et al. (2005) Malnutrition and associated factors among aged residents in all nursing homes in Helsinki. Eur J Clin Nutr 59, 578-583.

4. Elia M, Ritz P \& Stubbs RJ (2000) Total energy expenditure in the elderly. Eur J Clin Nutr 54, Suppl. 3, 40-47.

5. Crogan NL \& Pasvogel A (2003) The influence of proteincalorie malnutrition on quality of life in nursing homes. J Gerontol A Biol Sci Med Sci 58, M159-M164.

6. Sullivan DH \& Walls RC (1998) Protein-energy undernutrition and the risk of mortality within six years of hospital discharge. J Am Coll Nutr 17, 571-578.

7. Persson MD, Brismar KE, Katzarski KS, et al. (2002) Nutritional status using Mini Nutritional Assessment and Subjective Global Assessment predict mortality in geriatric patients. JAGS 50, 1996-2002.

8. Jongenelis K, Pot AM, Eisses AM, et al. (2004) Prevalence and risk indicators of depression in elderly nursing home patients: the AGED study. $J$ Affect Disord 83, 135-142.

9. Webber AP, Martin JL, Harker JO, et al. (2005) Depression in older patients admitted for postacute nursing home rehabilitation. J Am Geriatr Soc 53, 1017-1022.

10. Teresi J, Abrams R, Holmes D, et al. (2001) Prevalence of depression and depression recognition in nursing homes. Soc Psychiatry Psychiatr Epidemiol 36, 613-620.

11. Smalbrugge M, Pot AM, Jongenelis L, et al. (2006) The impact of depression and anxiety on well being, disability and use of health care services in nursing home patients. Int $J$ Geriatr Psychiatry 21, 325-332.

12. Rovner BW, German PS, Brant LJ, et al. (1991) Depression and mortality in nursing homes. JAMA 265, 994-996.

13. Morley JE \& Kraenzle D (1994) Causes of weight loss in a community nursing home. J Am Geriatr Soc 42, 583-585.

14. Blaum CS, Fries BE \& Fiatarone MA (1995) Factors associated with low body mass index and weight loss in nursing home residents. J Gerontol A Biol Sci Med Sci 50, M162-M168.

15. DiPietro L, Anda RF, Williamson DF, et al. (1992) Depressive symptoms and weight change in a national cohort of adults. Int $J$ Obes 16, 745-753.

16. German L, Feldblum I, Bilenko N, et al. (2008) Depressive symptoms and risk for malnutrition among hospitalized elderly people. Nutr Health Aging 12, 313-318.
17. Cabrera MAS, Mesas AE, Lopes Garcia AR, et al. (2007) Malnutrition and depression among community-dwelling elderly people. J Am Med Dir Assoc 8, 582-584.

18. Vellas B, Guigoz Y, Garry PJ, et al. (1999) The Mini Nutritional Assessment (MNA) and its use in grading the nutritional state of elderly patients. Nutrition 15, 116-122.

19. Chumlea WC, Roche AF \& Steinbauch ML (1985) Estimating stature from knee height for persons 60 to 90 years of age. $J$ Am Geriatr Soc 33, 116-120.

20. Sheikh JI \& Yesavage JA (1986) Recent evidence and development of a shorter version. Clinical Gerontology: A Guide to Assessment and Intervention, pp. 165-173 [TL Brink, editor]. New York: The Haworth Press.

21. O'Riordan TG, Hayes JP, O'Neill D, et al. (1990) The effect of mild to moderate dementia on the Geriatric Depression Scale and on the general health questionnaire. Age Ageing 19, 57-61.

22. Mahoney FI \& Barthel D (1965) Functional evaluation: the Barthel index. Md State Med J 14, 56-61.

23. Ware JE, Snow KK, Kosinski M, et al. (1993) SF-36 Health Survey: Manual and Interpretation Guide. Boston, MA: The Health Institute, New England Medical Center.

24. Moriguti JC, Das SK, Saltzman E, et al. (2000) Effects of a 6-week hypocaloric diet on changes in body composition, hunger, and subsequent weight regain in healthy young and older adults. J Gerontol A Biol Sci Med Sci 55, B580-B587.

25. Thomas P, Hazif-Thomas C \& Clement JP (2003) Influence of antidepressant therapies on weight and appetite in the elderly. J Nutr Health Aging 7, 166-170.

26. Abrams RC, Teresi JA \& Butin DN (1992) Depression in nursing home residents. Clin Geriatr Med 8, 309-322.

27. Skotzko CE, Krichten C, Zietowski G, et al. (2000) Depression is common and precludes accurate assessment of functional status in elderly patients with congestive heart failure. $J$ Card Fail 6, 300-305.

28. Alexopoulos GS, Vrontou C, Kakuma T, et al. (1996) Disability in geriatric depression. Am J Psychiatry 153, 877-885.

29. Teresi JA, Abrams R, Holmes D, et al. (2002) Influence of cognitive impairment, illness, gender, and African-American status on psychiatric ratings and staff recognition of depression. Am J Geriatr Psychiatry 10, 506-514.

30. Thakur M \& Blazer DG (2008) Depression in long-term care. J Am Med Dir Assoc 9, 82-87.

31. Payne JL, Sheppard JE, Steinberg M, et al. (2002) Incidence, prevalence, and outcomes of depression in residents of a longterm care facility with dementia. Int J Geriatr Psychiatry 17, $247-253$

32. Alexopoulos GS, Abrams RC, Young RC, et al. (1988) Cornell scale for depression in dementia. Biol Psychiatry 23, 271-284. 